\title{
Gesellschaftsbericht
}

\section{Forschungsförderungspreis 2003 der Croma Pharma GmbH}

Die Croma Pharma setzt einen Förderungspreis für eine bedeutende wissenschaftliche Arbeit auf dem Gebiet der Augenheilkunde in der Höhe von $€ \mathbf{3 . 5 0 0 , -}$ aus.

\section{Teilnahmebedingungen:}

1. Die Bewerber müssen das medizinische Hochschulstudium erfolgreich abgeschlossen haben, die Österreichische Staatsbürgerschaft besitzen und dürfen zum Zeitpunkt des Stichtages (siehe Punkt 2.) das 45. Lebensjahr noch nicht vollendet haben; sie dürfen weder Abteilungsleiter noch Klinikchef sein.

2. Die eingereichte Arbeit muss in Maschinschrift hergestellt sein; sie darf jedoch bereits innerhalb der letzten 2 Jahre publiziert worden sein. Die Arbeit muss bis spätestens

\section{März 2003}

bei der Österreichischen Ophthalmologischen Gesellschaft, Schlösselgasse 9, 1080 Wien, unter Hinweis auf die Bewerbung zum „Croma Pharma-Förderungspreis" in einem verschlossenen Umschlag einlangen. Der Arbeit ist ein kurzer Lebenslauf des Verfassers beizulegen. Die Einreichung wird vertraulich behandelt.
3. Über die Zuerkennung des Preises entscheidet eine Jury der Österreichischen Ophthalmologischen Gesellschaft. Bei gleichwertigen Arbeiten ist den praxisbezogenen der Vorzug zu geben.

4. Die Jury ist berechtigt, den Preis auf mehrere Verfasser zu verteilen; sie kann aber auch von der Zuerkennung des Preises absehen, wenn sie zur Überzeugung gelangt, dass keine preiswürdige Arbeit vorliegt.

5. Die Jury entscheidet mit einfacher Stimmenmehrheit. Ihre Entscheidung ist endgültig und unterliegt keinerlei Anfechtung, insbesondere auch nicht vor Gericht.

6. Die Croma Pharma ist berechtigt, den Inhalt der Arbeiten von Preisträgern im Rahmen der Aktivitäten der Croma Pharma zu verwerten, diese ganz - oder teilweise - zu veröffentlichen und Preisträger einzuladen, über das Thema ihrer Arbeit einen Vortrag zu halten.

7. Die gekürten Arbeiten werden im Spektrum der Augenheilkunde veröffentlicht.

8. Der Preis wird anlässlich der ÖOG in Salzburg, 29. bis 31. Mai 2003, von Herrn Mag. pharm. Gerhard Prinz, Gründer und Geschäftsführer der Croma Pharma, persönlich überreicht. 